\title{
Association of cytochrome P450 2 A6 polymorphism, anxiety, and environmental factors with cigarette smoking by Thai adults
}

Napakkawat Buathong ${ }^{\mathrm{a}}$, Rasmon Kalayasiri ${ }^{\mathrm{a}}$, Pajaree Chaliyavilaskul ${ }^{\mathrm{b}, \mathrm{c}}$, Khwanruan Phetnoi ${ }^{\mathrm{d}}$, Thanapoom Ratananuponge

${ }^{a}$ Department of Psychiatry, Faculty of Medicine, Chulalongkorn University, Bangkok 10330, Thailand ${ }^{b}$ Clinical Pharmacokinetics Research Unit in Renal and Cardiovascular Diseases, Faculty of Medicine, Chulalongkorn University, Bangkok 10330, Thailand

'Department of Pharmacology, Faculty of Medicine, Chulalongkorn University, Bangkok 10330, Thailand

${ }^{d}$ Department of Pharmacology, Graduate school, Chulalongkorn University, Bangkok 10330, Thailand ${ }^{e}$ Department of Preventive and Social Medicine, Faculty of Medicine, Chulalongkorn University, Bangkok 10330, Thailand

\begin{abstract}
Background: The effects and associations of genetic variation, psychological, and environment factors associated with cigarette smoking and nicotine dependence remain largely unknown.

Objective: To determine the influence and association of functional genetic polymorphisms of cytochrome P450 2A6 (CYP2A6), anxiety, and environmental factors on cigarette smoking and nicotine dependence.

Method: A cross-sectional study was conducted at King Chulalongkorn Memorial Hospital, Thailand between October 2014 and June 2015. We recruited 127 Thai adult smokers when they visited for an annual physical check-up. Participants completed questionnaires regarding demographic characteristics, The Fagerstr $\mathrm{m}$ Test for Nicotine Dependence, and The Thai Hospital Anxiety and Depression Scale. Blood was collected for CYP2A6 genotyping to determine the enzyme metabolism level/group.

Results: Factors associated with significantly greater cigarette consumption were age and being ultrarapid/ extensive metabolizers (UM/EM). Anxiety and smoking by household family members were significantly associated with the degree of nicotine dependence. We observed associations between severe nicotine dependence and genotype (UM/EM) and age $(b=0.037 ; P=0.005)$, intermediate metabolizers (IM) and age ( $b=0.031 ; P=0.43), \mathrm{UM} / \mathrm{EM}$ and anxiety $(b=0.258 ; P<0.001)$, IM and anxiety $(b=0.285 ; P<0.001), \mathrm{UM} / \mathrm{EM}$ and household smoking in the family members $(b=1.427 ; P=0.003)$, and IM and smoking by household family members $(b=1.293 ; P=0.024)$.

Conclusions: Information regarding the association between the gene encoding enzyme metabolism, anxiety, and their interactions may be beneficial for selecting treatment choices for smoking cessation for individual genotypic metabolizers.
\end{abstract}

Keywords: Anxiety, cigarette smoking, cytochrome P450 2A6 polymorphism

Cigarette smoking is a leading cause of morbidity and mortality worldwide. Several studies have shown that many factors including genetic variation, psychological, and environmental factors are associated with cigarette smoking and nicotine dependence [1].

Correspondence to: Napakkawat Buathong, Department of Psychiatry, Faculty of Medicine, Chulalongkorn University, Bangkok 10330, Thailand.E-mail: napakwawat.b@chula.ac.th
Genetic variation directly involved in the metabolism of drugs such as rs1051730 genetic variants in the nicotine acetylcholine receptor gene cluster (CHARNA5-CHARNA3-CHARNB4) and cytochrome P450s (CYPs). CYP2A6 is highly polymorphic with reduced function alleles leading to metabolic inactivation of nicotine and cotinine clearance [2-5]. Genetic polymorphisms of CYP2D6 affect cigarette consumption and nicotine dependence. Individuals with wild-type CYP2A6, classified as normal metabolizers, usually require more daily 
cigarettes than poor metabolizers, and were more likely to progress to nicotine dependence [6-9].

Psychological factors, especially anxiety and depression, are related to a greater level of smoking, cigarette consumption, and degree of nicotine dependence $[10,11]$. The relationships between anxiety, depression, cigarette consumption, and nicotine dependence have been explained either by nicotine intake causing an increase in the chances of developing anxiety and depression, or that anxiety and depression may induce smoking behavior [10, 12, 13]. The prevalence of cigarette consumption among persons with anxiety or mood disorders may be higher than individuals without psychiatric problems [14-16]. The finding from the Thai National Survey on mental health in 2008 reported that the prevalence of anxiety and major depressive disorder in the Thai population were $1.7 \%$ and $2.2 \%$ respectively. However, the prevalence of anxiety and major depressive disorders among Thais with illicit drug use and alcohol abuse were 1.8\%$16.5 \%$ and $1.3 \%-17.7 \%$, respectively [17]. Thus, the prevalence of anxiety and depression is increased in Thai populations with substance use. Other psychosocial factors may be related to cigarette use. The role of a smoking household member, or a peer or colleague smoking, have been demonstrated to be environmental factors that highly influence smoking behavior and nicotine dependence [18, 19]. However, to our knowledge, studies that determine the effects and associations of genetic variation, anxiety, and other environment factors on cigarette smoking are still largely lacking. Therefore, the objectives of this study were to determine the associations between genetic polymorphisms of CYP2A6, anxiety, and environment factors with cigarette consumption and nicotine dependence, and to examine the associations between genetic polymorphisms of CYP2A6 and anxiety, genetic polymorphisms of CYP2A6 and environmental and other related factors among Thai adult smokers.

\section{Materials and methods}

After approval by the Ethics Committee and the Institutional Review Board of the Faculty of Medicine, Chulalongkorn University (approval No. 367/2014), we conducted a cross-sectional study at King Chulalongkorn Memorial Hospital, Thailand between October 2014 and June 2015. We recruited 127 participants from Thai adult smokers who visited for a check-up at the Preventive and Social Medicine Clinic. The inclusion criteria were smokers who were willing to disclose their smoking behavior, aged from 18 to 60 years, having daily cigarette use and had smoked more than 100 cigarettes in the past 6 months. Exclusion criteria were subjects who had used any medication that may induce or inhibit CYP2A6 activity within 14 days of the study. The present study did not include pregnant women, or women with oral or injected contraception, or individuals with kidney or liver disease. All participants provided their written informed consent to participate.

All participants completed questionnaires to determine demographic data, smoking behavior in the past month (to control recall bias), environmental factors regarding smoking household members and colleagues, The Fagerstr $m$ Test for Nicotine Dependence, and The Thai Hospital Anxiety and Depression Scale for evaluation of anxiety and depression. The Fagerstr $m$ Test for Nicotine Dependence $[20,21]$ is a widely used 6 item selfreport instrument to measure the degree of nicotine dependence. The total scores range from $0-10$, with higher scores indicating a higher degree of dependence. In this study, the scores $0-3,4-5$, and 6-10 indicated low, moderate, and high dependence status, respectively. The Thai Hospital Anxiety and Depression Scale (The Thai-HADS), a reliable and valid instrument for screening anxiety and depression in both Thai patients and general populations were applied [22-24]. The test is composed of 14 items (each item scored 0-3). Seven items relate to depressive symptoms and the rest of 7 items relate to anxiety symptoms. A cut-off point of $\geq 11$ was interpreted as a clinical case of both depression and anxiety. The sensitivity of anxiety and depression subscales of the Thai HADS are $100 \%$ and $85.71 \%$, respectively, while the specificity is $86.0 \%$ for anxiety, and $91.3 \%$ for depression. Both subscales also showed good internal consistencies with a Cronbach's alpha coefficient of 0.85 for the anxiety subscale and 0.83 for the depression subscale.

\section{CYP2A6 genotyping}

Blood samples were collected into ethylenediaminetetraacetic acid containing tubes and transported on dry ice to a $-80^{\circ} \mathrm{C}$ freezer within $3 \mathrm{~h}$ of collection. Genomic DNA was extracted using a Purelink Genomic DNA mini kit (Invitrogen, USA). The genotyping of CYP2A $6 * 9$ alleles was assessed using real-time PCR with Stepone software, version 2.2, and an Applied Biosystems 7500 Real Time PCR System. 
The genotyping of CYP2A6*1A, CYP2A6*1B, and CYP2A6*4 alleles were determined using a restriction fragment length polymorphism method (PCR-RFLP) [25].

CYP2A6 genotypes were categorized into 4 groups: ultrarapid metabolizers (UM) including persons with more than 2 CYP2A6*1x2A or CYP2A61x2B alleles; extensive metabolizers (EM) included those having 2 CYP2A6* $1 \mathrm{~A} / \mathrm{x} 1 \mathrm{~A}$ or CYP2A6* $1 \mathrm{~A} / \mathrm{x} 1 \mathrm{~B}$ or CYP2A6* $1 \mathrm{~A} / \mathrm{x} 1 \mathrm{~B}$ alleles; intermediate metabolizers (IM) included those having either one CYP2A6*1A/ $\mathrm{x} 4 \mathrm{C}, \mathrm{CYP} 2 \mathrm{~A} 6 * 1 \mathrm{~B} / \mathrm{x} 4 \mathrm{C}, \mathrm{CYP} 2 \mathrm{~A} 6 * 1 \mathrm{~A} / \mathrm{x} * 9$, or CYP2A6*1B/X*9; and poor metabolizers $(\mathrm{PM})$ including participants having 2 copies of the inactive variants (CYP2A6*4C) or having one or 2 CYP2A6*4C/x*9, CYP2A6*9A/x10 variants [9].

\section{Statistical analysis}

The frequency of categorical variables was analyzed using descriptive variables. Mean and standard deviation (SD) were used to describe continuous variables. Univariate analysis for associations of independent variables with nicotine dependence and cigarette consumption were analyzed using an independent sample $t$ test, one-way analysis of variance (one-way ANOVA), Kruskal-Wallis test, and Spearman correlation. Multiple linear regressions were used to examine factors predicting nicotine dependence and cigarette consumption. The associations were also estimated in multiple linear regression to examine the effect between genotype and age, genotype and anxiety, and CYP2A6 metabolizers genotype and environment factors on the degree of nicotine dependence (score) and amount of cigarette consumption. All data analyses were conducted using STATA, version 11.0 (STATA Corp, College Station, Texas).

\section{Results}

We recruited 127 Thai adult smokers (96\% men) with a mean age $(\mathrm{SD})=37.5$ (9.99) years, range 1859 years. Baseline data regarding socioeconomic status are presented in Table 1. The prevalence of individuals with low, moderate, and high nicotine dependence were $46 \%, 22 \%$, and $32 \%$, respectively. On average, participants were moderately nicotine dependent with a Fagerstr $m$ Test for Nicotine Dependence score of 3.69 (2.56). The mean age (SD) at which participants starting smoking was 17.0 (3.82) years (range 9-35 years); the mean duration (SD) of their smoking was 20.5 (9.99) years (range 1-44 years); the mean (SD) quantity of cigarette consumption was 11.86 (6.86) per day (range 1-40 cigarettes per day). Table 1 presents a comparison of the demographics of participants in relation to daily cigarette consumption and nicotine dependence. Being female was significantly associated with higher nicotine dependence. By contrast, education, marital status, occupation, alcohol, and caffeine consumption were not associated with amount of cigarette smoking or severity of nicotine dependence $(P>0.05)$.

\section{CYP2A6 genotype, anxiety, depression, environmental and related factors according to daily cigarette consumption and nicotine dependence}

Prevalence of anxiety was $13 \%$ and depression was $9 \%$ among adult smokers. Most participants exposed to a household member smoking (85\%) or colleague smoking (94\%) in their environment.

Based on CYP2A6 genotype, there were UM (28\%); (mean (SD) cigarette consumption $=13.14$ (7.97)), EM (32.3\%); (mean (SD) cigarette consumption = $13.02(7.65))$, IM (27.6\%); $($ mean cigarette consumption 10.77 (4.71)) and PM (12.6\%); (mean cigarette consumption $=8.50(4.66)$ ). . Because the average cigarette consumption per day between UM and EM groups were not different, UM and EM were combined for inferential statistical analysis.

Table 2 presents comparisons of the means of CYP2A6 genotype, anxiety, depression, environmental factors, and smoking information according to daily cigarette consumption and nicotine dependence. We found differences in the average number of cigarette consumed between each type of CYP2A6 genotype. A post hoc comparison test indicated that participants with UM/EM smoked a greater number of cigarettes per day. This difference from participants who are poor metabolizers was significant. However, we found that there were no significant differences in the degree of nicotine dependence between each CYP2A6 genotype. Anxiety was not associated with cigarette consumption, but was associated with the degree of nicotine dependence. Likewise, a household member smoking was not significantly associated with cigarette consumption, but was statistically associated with degree of nicotine dependence.

Table 3 demonstrates the correlations between continuous variables and cigarette consumption and nicotine dependence. Age and duration of smoking 
were positively correlated with cigarette consumption. Positive correlations between age, duration of smoking, and anxiety score were found with nicotine dependence. The multicollinearity between age and duration of smoking habit were seen. Age was then selected into the multiple regression analysis.

Table 1. Comparisons of demographic characteristics according to daily cigarette consumption and nicotine dependence (Fagerstr m Test for Nicotine Dependence score)

\begin{tabular}{|c|c|c|c|c|c|c|c|c|c|c|c|}
\hline & \multicolumn{6}{|c|}{ Cigarette consumption } & \multicolumn{5}{|c|}{ Nicotine dependence } \\
\hline & $\bar{n}$ & Mean & SD & Statistic ( & (df) & $\dagger P$ & Mean & SD & Statistic (c & & $\dagger P$ \\
\hline \multicolumn{12}{|l|}{ Sex } \\
\hline Male & 122 & 11.8 & 6.87 & $t(125)$ & -0.64 & 0.522 & 3.6 & 2.58 & $t(7.18)$ & -3.83 & 0.006 \\
\hline Female & 5 & 13.8 & 6.87 & & & & 5.4 & 0.89 & & & \\
\hline \multicolumn{12}{|l|}{ Education } \\
\hline Primary school & 31 & 14.4 & 9.39 & $\chi^{2}$ & 2.11 & 0.347 & 4.2 & 2.87 & $F(2,124)$ & 1.77 & 0.18 \\
\hline Secondary/vocational & 77 & 11.0 & 5.84 & & & & 3.4 & 2.54 & & & \\
\hline Bachelor’sdegree/higher & 19 & 11.4 & 4.85 & & & & 4.2 & 1.93 & & & \\
\hline \multicolumn{12}{|l|}{ Marital status } \\
\hline Single/divorced/widowed & 65 & 11.6 & 5.84 & $t(112.69)$ & -0.42 & 0.677 & 3.7 & 2.53 & $t(125)$ & 0.07 & 0.95 \\
\hline Married & 62 & 12.1 & 7.82 & & & & 3.7 & 2.61 & & & \\
\hline \multicolumn{12}{|l|}{ Employment } \\
\hline Employee/ laborer & 108 & 12.3 & 7.01 & $F(2,124)$ & 1.67 & 0.193 & 3.6 & 2.53 & $F(2,124)$ & 0.30 & 0.74 \\
\hline Government and state enterprise & 10 & 10.0 & 5.03 & & & & 4.3 & 2.87 & & & \\
\hline Own business & 9 & 8.6 & 5.96 & & & & 3.7 & 2.79 & & & \\
\hline \multicolumn{12}{|l|}{ Salary (baht) } \\
\hline$\leq 10,000$ & 50 & 13.1 & 7.22 & $F(2,124)$ & 1.65 & 0.196 & 4.1 & 2.64 & $F(2,124)$ & 2.95 & 0.06 \\
\hline $10,001-15,000$ & 36 & 10.4 & 6.05 & & & & 2.8 & 2.47 & & & \\
\hline$>15,000$ & 41 & 11.7 & 6.95 & & & & 4.0 & 2.43 & & & \\
\hline \multicolumn{12}{|l|}{ Alcohol consumption } \\
\hline No & 37 & 13.6 & 6.92 & $t(125)$ & 1.84 & 0.068 & 4.1 & 2.35 & $t(125)$ & 1.25 & 0.21 \\
\hline Yes & 90 & 11.2 & 6.74 & & & & 3.5 & 2.64 & & & \\
\hline \multicolumn{12}{|l|}{ Caffeine consumption } \\
\hline No & 33 & 11.2 & 5.75 & $t(125)$ & -0.64 & 0.526 & 3.6 & 2.81 & $t(125)$ & -0.15 & 0.88 \\
\hline Yes & 94 & 12.1 & 7.22 & & & & 3.7 & 2.48 & & & \\
\hline
\end{tabular}

$\mathrm{df}=$ Degrees of freedom. $\downarrow P$ was based on an independent sample $t$ test, one-way ANOVA, or Kruskal-Wallis test as appropriate.

Table 2. CYP2A6 genotype, anxiety, depression, environmental factors according to daily amount of cigarette consumption and severity of nicotine dependence

\begin{tabular}{|c|c|c|c|c|c|c|c|c|c|c|c|}
\hline & \multicolumn{6}{|c|}{ Cigarette consumption } & \multicolumn{5}{|c|}{ Nicotine dependence } \\
\hline & $\bar{n}$ & Mean & SD & Statistic & (df) & $\dagger P$ & Mean & SD & Statistic & df) & $\dagger P$ \\
\hline No & 19 & 9.8 & 5.29 & $t(125)$ & -1.40 & 0.164 & 2.2 & 2.25 & $t(125)$ & -2.81 & 0.006 \\
\hline Yes & 108 & 12.2 & 7.06 & & & & 4.0 & 2.53 & & & \\
\hline \multicolumn{12}{|c|}{ Smoking colleague } \\
\hline No & 10 & 15.2 & 10.35 & $t(9.61)$ & 1.09 & 0.303 & 4.1 & 2.64 & $t(125)$ & 0.52 & 0.60 \\
\hline Yes & 117 & 11.6 & 6.46 & & & & 3.7 & 2.56 & & & \\
\hline \multicolumn{12}{|l|}{ Anxiety } \\
\hline No & 111 & 11.6 & 6.46 & $t(17.22)$ & -1.00 & 0.331 & 3.4 & 2.46 & $t(125)$ & -3.96 & $<0.001$ \\
\hline Yes & 16 & 13.9 & 9.15 & & & & 5.9 & 2.11 & & & \\
\hline \multicolumn{12}{|c|}{ Depression } \\
\hline No & 116 & 12.2 & 6.88 & $t(125)$ & 1.98 & 0.05 & 3.7 & 2.56 & $t(125)$ & -0.17 & 0.87 \\
\hline Yes & 11 & 8.0 & 5.42 & & & & 3.8 & 2.75 & & & \\
\hline \multicolumn{12}{|c|}{ CYP2A6 genotype } \\
\hline UM/EM & 76 & 13.1 & 7.75 & c2 & 6.09 & 0.048 & 3.9 & 2.53 & $F(2,124)$ & 2.11 & 0.13 \\
\hline IM & 35 & 10.8 & 4.72 & & & & 3.7 & 2.66 & & & \\
\hline PM & 16 & 8.5 & 4.66 & & & & 2.5 & 2.28 & & & \\
\hline
\end{tabular}

$\mathrm{df}=$ Degrees of freedom. $\downarrow P$ were based on independent sample $t$ test, one-way ANOVA or Kruskal-Wallis test as appropriate. UM/EM, ultrarapid/extensive metabolizers. 
Table 3. Means, standard deviation, and bivariate correlation of the study variables

\begin{tabular}{|c|c|c|c|c|c|c|c|c|}
\hline & Mean & SD & $\begin{array}{c}1 \\
\text { Age }\end{array}$ & $\begin{array}{c}2 \\
\text { Duration of } \\
\text { smoking habit }\end{array}$ & $\begin{array}{c}3 \\
\text { Anxiety }\end{array}$ & $\begin{array}{c}4 \\
\text { Depression }\end{array}$ & $\begin{array}{c}5 \\
\text { Cigarette } \\
\text { consumption }\end{array}$ & $\begin{array}{c}6 \\
\text { Nicotine } \\
\text { dependence }\end{array}$ \\
\hline 1. Age & 37.5 & 9.99 & - & & & & & \\
\hline $\begin{array}{l}\text { 2. Duration of } \\
\text { smoke (years) }\end{array}$ & 20.5 & 9.98 & $\begin{array}{l}r=0.93 \\
P<0.001\end{array}$ & - & & & & \\
\hline 3. Anxiety & 6.5 & 3.43 & $\begin{array}{l}r=0.15 \\
P=0.09\end{array}$ & $\begin{array}{l}r=0.08 \\
P=0.36\end{array}$ & - & & & \\
\hline 4. Depression & 5.1 & 3.45 & $\begin{array}{l}r=0.11 \\
P=0.23\end{array}$ & $\begin{array}{l}r=0.04 \\
P=0.65\end{array}$ & $\begin{array}{l}r=0.42 \\
P<0.001\end{array}$ & - & & \\
\hline $\begin{array}{l}\text { 5. Cigarette } \\
\text { consumption }\end{array}$ & 11.9 & 2.56 & $\begin{array}{l}r=0.22 \\
P=0.01\end{array}$ & $\begin{array}{l}r=0.22 \\
P=0.013\end{array}$ & $\begin{array}{l}r=0.13 \\
P=0.14\end{array}$ & $\begin{array}{l}r=-0.17 \\
P=0.06\end{array}$ & - & \\
\hline $\begin{array}{l}\text { 6. Nicotine } \\
\text { dependence }\end{array}$ & 3.7 & 2.56 & $\begin{array}{l}r=0.241 \\
P=0.006\end{array}$ & $\begin{array}{l}r=0.23 \\
P=0.009\end{array}$ & $\begin{array}{l}r=0.47 \\
P<0.001\end{array}$ & $\begin{array}{l}r=0.08 \\
P=0.36\end{array}$ & $\begin{array}{l}r=0.63 \\
P<0.001\end{array}$ & - \\
\hline
\end{tabular}

*Bivariate correlation analysis was performed using a Spearman correlation

Predictors of cigarette consumption and nicotine dependence

Table 4 shows predictors for cigarette consumption and degree of nicotine dependence. We analyzed the associations between gene and age, gene and anxiety, and gene and environmental factors on the degree of nicotine dependence and on cigarette consumption upon different adjustments of covariate, and using genetically poor metabolizers as a reference group.

In model 1, the predictors for cigarette consumption were age and UM/EM. Older age was significantly associated with greater cigarette consumption. UM/EM was also associated with greater cigarette consumption. Model 2 presented the associations of gene and anxiety on cigarette consumption after adjusting for sex, age, and a smoking household member. UM/EM and anxiety were associated with greater cigarette consumption. In Model 3, after adjusting for sex, age and anxiety, $\mathrm{UM} / \mathrm{EM}$, and a smoking household member, were associated with greater cigarette consumption. In Model 4, after adjusting for sex, a smoking household member, and anxiety, UM/EM, and age were associated with greater cigarette consumption.

In Model 1, the predictors for a greater degree of nicotine dependence score were age, anxiety, and a smoking household member. By contrast, metabolizer groups were not significantly associated with the degree of nicotine dependence. However, in Model 2 where the associations of genes and anxiety on the degree of nicotine dependence were examined, after adjusting for sex, age, and smoking household member, $\mathrm{UM} / \mathrm{EM}$ and anxiety, and IM and anxiety were associated with a greater nicotine dependence score. In Model 3, after adjusting for sex, age, and anxiety, UM/EM and smoking household member, and IM and smoking household member were associated with an increased in nicotine dependence score. In Model 4, after adjusting for sex, smoking household member, and anxiety, UM/EM and age were associated with a greater degree of nicotine dependence indicated by a higher nicotine dependence score.

\section{Discussion}

We found that the proportion of UM/EM in participants was $60 \%$. This is higher than that of the previous study in Thailand, where the prevalence of UM/EM group in Thai smokers was 37.5\% [26]. This higher proportion of UM/EM in Thai Adult smokers in our study probably reflects that all of our participants were current smokers, while data of the previous study was collected on general population. Associations between UM/EM and cigarette consumption were found. This finding is consistent with a previous study that found that the nicotine and cotinine clearance in $\mathrm{UM} / \mathrm{EM}$ smokers is faster than that by IM or PM and might lead to higher cigarette consumption [6-9, 27]. However, CYP2A6 polymorphisms were not a predictor of the degree of nicotine dependence.

Previous studies described that female sex is associated with a higher rate of nicotine biotransformation compared with male sex, leading to higher rate of withdrawal symptoms [28, 29]. Consistent with this, our study found that female smokers had an average degree of nicotine dependence score that was higher than that for male smokers. 
Table 4. Predictors of cigarette consumption and nicotine dependence

\begin{tabular}{|c|c|c|c|c|c|c|c|c|}
\hline & \multicolumn{4}{|c|}{ Cigarette consumption } & \multicolumn{4}{|c|}{ Nicotine dependence } \\
\hline & $b$ & $\mathrm{SE}(b)$ & $95 \% \mathrm{CI}(b)$ & $\boldsymbol{P}$ & $\boldsymbol{b}$ & $\mathrm{SE}(b)$ & $95 \% \mathrm{CI}(b)$ & $\boldsymbol{P}$ \\
\hline \multicolumn{9}{|l|}{ Model 1} \\
\hline Female sex & -0.626 & 3.057 & $-6.68,5.43$ & 0.838 & 0.756 & 1.022 & $-1.27,2.78$ & 0.461 \\
\hline Age $^{a}$ & 0.155 & 0.061 & $0.03,0.276$ & 0.012 & 0.036 & 0.020 & $-0.005,0.08$ & 0.082 \\
\hline Smoking household member ${ }^{\mathrm{b}}$ & 2.047 & 1.669 & $-1.26,5.35$ & 0.222 & 1.208 & 0.558 & $0.10,2.31$ & 0.032 \\
\hline Anxiety & 0.168 & 0.177 & $-0.18,0.52$ & 0.344 & 0.309 & 0.059 & $0.19,0.43$ & $<0.001$ \\
\hline $\mathrm{UM} / \mathrm{EM}^{\mathrm{c}}$ & 3.929 & 1.821 & $0.32,7.53$ & 0.033 & 0.977 & 0.609 & $-0.23,2.18$ & 0.111 \\
\hline $\mathrm{IM}^{\mathrm{c}}$ & 2.127 & 1.988 & $-1.81,6.06$ & 0.287 & 0.970 & 0.665 & $-0.35,2.29$ & 0.147 \\
\hline \multicolumn{9}{|l|}{ Model $2^{\mathrm{d}}$} \\
\hline Anxiety and UM/EM ${ }^{c}$ & 0.363 & 0.167 & $0.03,0.69$ & 0.031 & 0.258 & 0.057 & $0.15,0.37$ & $<0.001$ \\
\hline Anxiety and $\mathrm{IM}^{\mathrm{c}}$ & 0.049 & 0.20 & $-0.35,0.44$ & 0.808 & 0.285 & 0.068 & $0.15,0.42$ & $<0.001$ \\
\hline \multicolumn{9}{|l|}{ Model $3^{e}$} \\
\hline \multicolumn{9}{|l|}{ Smoking household member } \\
\hline Smoking and UM/EM ${ }^{c}$ & 4.127 & 1.408 & $1.34,6.14$ & 0.004 & 1.427 & 0.471 & $0.49,2.36$ & 0.003 \\
\hline \multicolumn{9}{|l|}{ Smoking household member } \\
\hline Smoking and $\mathrm{IM}^{\mathrm{c}}$ & 1.574 & 1.686 & $-1.76,4.91$ & 0.352 & 1.293 & 0.563 & $0.18,2.41$ & 0.024 \\
\hline \multicolumn{9}{|l|}{ Model $4^{\mathrm{f}}$} \\
\hline Age and UM/EM ${ }^{c}$ & 0.149 & 0.039 & $0.07,0.23$ & $<0.001$ & 0.037 & 0.013 & $0.01,0.06$ & 0.005 \\
\hline Age and $\mathrm{IM}^{\mathrm{c}}$ & 0.082 & 0.046 & $-0.01,0.17$ & 0.071 & 0.031 & 0.015 & $0.001,0.06$ & 0.043 \\
\hline
\end{tabular}

${ }^{\text {a} A g e ~ a n d ~ a n x i e t y ~ v a r i a b l e s ~ a s ~ a ~ c o n t i n u o u s ~ d a t a ~ i n t o ~ t h e ~ m o d e l . ~}$

bDummy variable (reference with “no”)

'Dummy variable (reference with "Poor metabolizers")

${ }^{\mathrm{d}}$ Model 2 was adjusted for gender, age, and smoking household member.

${ }^{\mathrm{e}}$ Model 3 was adjusted for gender, age, and anxiety.

${ }^{\mathrm{f}}$ Model 4 was adjusted for gender, smoking household member, and anxiety.

UM/EM, ultrarapid/extensive metabolizers; IM, intermediate metabolizers.

To our knowledge, the associations between UM/ EM and age, anxiety, and environmental factors were associated with cigarette consumption and nicotine dependence. The effect of the associations between $\mathrm{UM} / \mathrm{EM}$ and a smoking household member was the strongest predictor for greater cigarette consumption and the degree of nicotine dependence. This might be the consequence of genetic variations and the individual habits of smokers in their family environment. Environmental factors highly influence the initiation of smoking by adolescents and lead to persistent smoking and resulting nicotine dependence $[19,30]$. Determining associations between genetic variation and environmental factors is important knowledge for health care professionals in all areas. Prevention of cigarette smoking in a home environment can reduce the number of new smokers and might also be a strategies for smokers who are attempting to quit smoking. Apart from environmental factors, the associations between UM/EM and anxiety were also associated with cigarette use, as similarly found in a previous study that described associations between anxiety and nicotine dependence. The relationship between anxiety, depression, cigarette consumption, and nicotine dependence were studied as co-occurring mental health disorders. Anxiety and depression may be associated with an increase in cigarette consumption as a self-medicating behavior in order to cope with their stress $[10,12,13]$. Moreover, one study found that cigarette consumption may play a role in the onset of mental illness [31].

Information on the association of genes, anxiety, and their interactions may be of benefit for selecting available treatments in smoking cessation programs in regard to individual CYP2A6 genotypes. A urine testing method to determine CYP2A6 genotypes may be practical for physicians in the clinic in considering the benefit of various treatments for smokers. Nonetheless, health care providers should still consider the important role of psychological counseling, relaxation techniques, or cognitive behavior therapy to help their smoking patients to cope with anxiety associated with nicotine dependence.

Our participants were current smokers who visited for their annual medical check-up. This means they might have a physical or health status that differs from 
smokers in other settings. The relatively small sample size may also have led to difficulty in identifying some predictors of cigarette use. In addition, we used a candidate gene approach in this study and only one gene was selected. However, we might be able to generalize the results of the association between their CYP2A6 genotype and cigarette smoking to the Thai population. A further study with more intensive genotyping including genome-wide associations study may provide better insight into the influence of genetic variation on nicotine use and dependence.

\section{Acknowledgment}

This study was supported by Rachadapiseksompotch Fund, Faculty of Medicine, Chulalongkorn University (RA57/110). We thank all of the nurses and staff at the Preventive and Social Medicine Clinic for their valuable assistance. Rasmon Kalayasiri was supported in a research career by the Fogarty International Center of the National Institutes of Health (NIH) under award number D43TW09087. Khwanruan Phetnoi was supported by the $90^{\text {th }}$ Anniversary of Chulalongkorn University (GCUGR $1125582041 \mathrm{M})$.

\section{Conflict of interest statement}

The authors declare that there is no conflict of interest in this research.

\section{References}

1. Schmidt A, Neumann M, Writz M, Errnstmann N, Straatschek-Jok A, Stroelben E, et al. The influence of occupational stress factors on the nicotine dependence: a cross sectional study. Tob Induc Dis. 2010; 8:1-6.

2. Caporoso N, Gu F, Sheng-Chih J, Yu K, Yeager M, Chan C, et al. Genome-wide and candidate gene association study of cigarette smoking behaviors. PLoS One. 2009; 4:e4653.

3. Thorgeirsson TE, Gudbjartsson DF, Surakka I, Vink JM, Amin N, Geller F, et al. Sequence variants at CHRNB3-CHRNA6 and CYP2A6 affecting smoking behavior. Nature Genetics. 2010; 42:448-53.

4. Nelson DR, Koymans T, Stegeman JJ, Fey-ereisen R, Waxman DJ, et al. P450 superfamily: update on new sequences, gene mapping, accession numbers and nomenclature. Pharmacogenetics. 1996; 6:1-42.

5. Nakajima M, Yamamoto T, Nunoya KI, Yokoi T, Nagashima K, Inoue K, et al. Role of human cytochrome P4502A6 in C-oxidation of nicotine. Drug metab Dispos. 1996; 24:1212-17.

6. Malaiyandi V, Lerman C, Benowitz, NL, Jepson C, Patterson F, Tyndale R.F. Impact of CYP2A6 genotype on pretreatment smoking behavior and nicotine levels from and usage of nicotine replacement therapy. Mol Psychiatry. 2006; 11:400-9.

7. Audrain-McGovern J, Koudsi N.A, Rodriguez D, Wileyto, EP, Shields PG. The role of CYP2A6 in the emergence of nicotine dependence in adolescents. Pediatrics. 2007; 119:e264-74.

8. Benowitz NL, Perez-Stable EL, Herrer, B, Jacob P. Slower metabolism and reduced intake of nicotine from cigarette smoking in Chinese-Americans. J Natl Cancer Inst. 2002; 94:108-15.

9. Liu T, David S P, Tyndale RF, Wang H, Zhou Q, Ding $\mathrm{P}$, et al. Associations of CYP2A6 genotype with smoking behaviors in southern China. Addiction. 2011; 106:985-94.

10. Moylan S, Jacka FN, Pasco JA, Berg, M. Cigarette smoking, nicotine dependence and anxiety disorder : a systematic review of population-based, epidemiology studies. BMC Medicine. 2012; 10:123.

11. Lawrence D, Considine J, Mitrou F, Zubrick S.R. Anxiety disorders and cigarette smoking: Results from the Australian Survey of Mental health and Wellbeing. Aust NZ Psychiatry. 2010; 44:520-7.

12. John U, Meyer C, Phil HR, Phil UH. Depressive disorders are related to nicotine dependence in the population but do not necessarily hamper smoking cessation. J Clin Psychiatry. 2004; 6:169-76.

13. Jamal M, Willem Van Der Does AJ, Cuijpers P, Penninx BW. Association of smoking and nicotine dependence with severity and course of symptoms in patients with depressive or anxiety disorder. Drug Alcohol Depend. 2012; 126:138-46.

14. Goodwin RD, Zvolensky MJ, Keyes, KM. Nicotine dependence and mental disorders among adults in the USA: Evaluating the role of the mode of administration. Psychol Med. 2008; 38:1277-86.

15. Grover KW, Goodwin RD, Zvolensky MJ. Does current versus former smoking play role in the relationship between anxiety and mood disorders and nicotine dependence? Addict Behav. 2012; 37:682-5.

16. Weinberger AH, Desai RA, McKee SA. Nicotine withdrawal in U.S. smokers with current mood, anxiety, alcohol use, and substance use disorders. Drug Alcohol Depend. 2010; 108:7-12.

17. Suttajit S, Kittirattanapaiboon P, Junsirimongkol B, Likhitsatian S, Srisurapanont M. Risks of major depressive disorder and anxiety disorders among 
Thais with alcohol use disorders and illicit drugs use: Finding from the 2008 Thai National Mental Health survey. Addict Behav. 2012; 37:1395-9.

18. Brook JS, Saar N, Zhang C, Brook DA. Familial and nonfamilial smoking: effects on smoking and nicotine dependence. Drug Alcohol Depend. 2009; 101:62-8.

19. Tully EC, Iacono, WG, McGue M. Changes in genetic and environmental influences on the development of nicotine dependence and major depressive disorder from middle adolescence to early adulthood. Dev Psychopathol. 2010; 22:831-48.

20. Heatherton TF, Kozlowski LT, Frecker RC, Fagerstr m KO. The Fagerstr $\mathrm{m}$ Test for Nicotine Dependence: a revision of the Fagerstr m Tolerance Questionnaire. Br J Addict. 1991; 86:1119-27.

21. Pomerleau CS, Carton SM, Lutzke ML, Flessland, KA, Pomerleau OF. Reliability of the Fagerstrom Tolerance Questionnaire and the Fagerstrom Test for Nicotine Dependence. Addict Behav. 1994; 19:33-9.

22. Nilchaikovit T, Lotrakul M, Phisansuthideth U. Development of Thai version of Hospital anxiety and depression scale in cancer patients. J Thai Psych Assoc. 1996; 41:18-30.

23. Zigmond AS, Snaith RP. The hospital anxiety and depression scale. Acta Psychiatr Scand. 1983; 67: 361-70.

24. Bjelland I, Dahl AA, Haug TT, Neckelmann D. The validity of the hospital anxiety and depression scale. An updated literature review. Psychosom Res. 2002; 52:69-77.

25. Ariyoshi N, Takahashi Y, Miyamoto M, Umetsu Y, Daigo S, Tateishi T, et al. Structural characterization of a new variant of the CYP2A6 gene (CYP2A6*1B) apparently diagnosed as heterozygotes of CYP2A6*1A and CYP2A6*4C. Pharmacogenetics. 2000; 10:687-93.

26. Apinan R, Tassaneeyakul W, Satarug S, Kajanawart S, Vannaprasaht S, Ruenweerayut R, et al. The influence of CYP2A6 polymorphisms and cadmium on nicotine metabolism in Thai population. Environ Toxicol Pharmacol. 2010; 28:420-24.

27. Mahavorasirikul W, Tassaneeyakul W, Satarug S, Ruengweerawut R, Na-bangchang C, Na-bangchang K. CYP2A6 genotypes and coumarin-oxidation phenotypes in a Thai population and their relationship to tobacco smoking. Eur J Clin Pharmacol. 2009; 65: 377-84.

28. Benowitz NL, Lessov-Schlaggar CN, Swan GE, Jacob III P. Female sex and oral contraceptive use accelerate nicotine metabolism. Clin Pharmacol Ther. 2006; 79: 480-88.

29. Rubinstein ML, Benowitz NL, Auerback GM, Moscicki AB. Rate of nicotine metabolism and withdrawal symptoms in adolescent light smokers. Pediatrics. 2008; 122:e643-47.

30. Fergusson DM, Horwood LJ, Boden JM, Jenkin G. Childhood social disadvantage and smoking in adulthood: results of a 25-year longitudinal study. Addiction. 2007; 102:475-82.

31. Cuijpers P, Smit F, ten Have M, de Graaf R. Smoking is associated with first-ever incidence of mental disorders: a prospective population-based study. Addiction. 2007; 102:1303-9. 